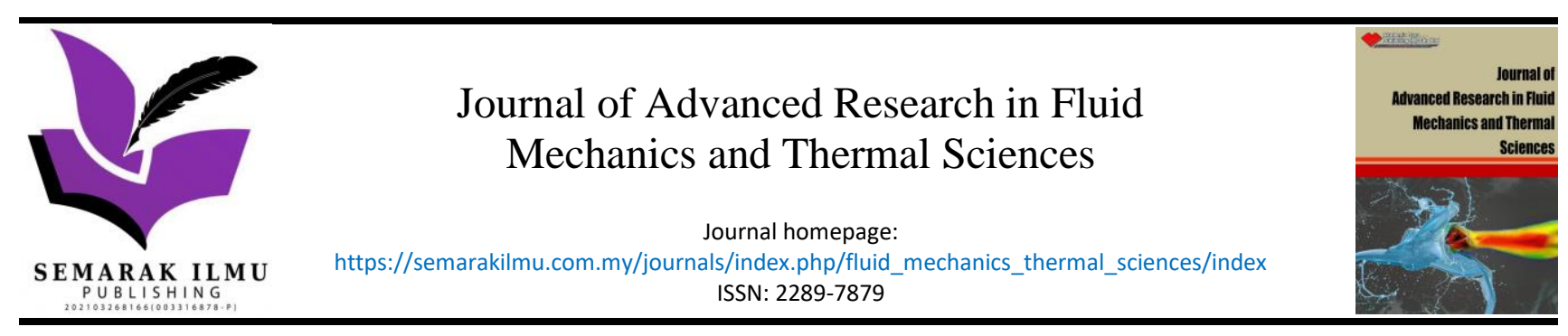

\title{
Flow and Heat Transfer of MHD Dusty Nanofluid Toward Moving Plate with Convective Boundary Condition
}

\author{
Euwing Low ${ }^{1, *}$, Syahira Mansur ${ }^{1, *}$, Yaan Yee Choy ${ }^{1}$, Eugene Low ${ }^{2}$ \\ 1 Faculty of Applied Sciences and Technology, Universiti Tun Hussein Onn Malaysia (Pagoh Campus), 84600 Pagoh, Johor, Malaysia \\ 2 Faculty of Electrical and Electronic Engineering, Universiti Tun Hussein Onn Malaysia, 86400 Parit Raja, Batu Pahat, Johor, Malaysia
}

\begin{tabular}{ll} 
ARTICLE INFO & ABSTRACT \\
\hline $\begin{array}{l}\text { Article history: } \\
\text { Received } 9 \text { June } 2021 \\
\text { Received in revised form } 25 \text { October 2021 } \\
\text { Accepted } 29 \text { October } 2021\end{array}$ & $\begin{array}{l}\text { This paper considers the flow and heat transfer characteristics of dusty nanofluid over a } \\
\text { moving plate in the presence of magnetohydrodynamic (MHD) with convective boundary } \\
\text { condition. Two types of nanofluid namely CuO-water and } \mathrm{Al}_{2} \mathrm{O}_{3} \text {-water permeated with } \\
\text { dust particles are considered. The governing partial differential equations are converted } \\
\text { into a system of non-linear ordinary differential equations using similarity transformation, } \\
\text { then the non-linear ordinary differential equations are solved using shooting method with } \\
\text { fourth-fifth order Runge-Kutta Fehlberg method (RKF45). The influence of non- } \\
\text { dimensional governing parameters such as velocity ratio parameter, magnetic field } \\
\text { parameter, volume fraction of the nanoparticle, volume fraction of the dust particle, mass } \\
\text { concentration of the dust particle, fluid particle interaction parameter for velocity, fluid } \\
\text { particle interaction parameter for temperature and Biot number on the velocity and } \\
\text { temperature profiles for fluid and dust phases of CuO-water and } \mathrm{Al}_{2} \mathrm{O}_{3} \text {-water dusty } \\
\text { nanofluids are discussed and presented through graphs. The skin friction coefficient and } \\
\text { Nusselt number are discussed and presented in tabular form. }\end{array}$ \\
$\begin{array}{l}\text { Keywords: } \\
\text { MHD; Dusty fluid; Nanofluid; Moving }\end{array}$ \\
plate; Convective boundary condition
\end{tabular}

\section{Introduction}

The fluid flow and heat transfer over a moving plate have a range of applications in applied science and engineering areas such as transportation, nuclear reactors, biomedicine and electronics. In present times, the convectional heat transfer fluid suffered different issues in engineering electronic devices due to relatively low thermal conductivity. Thus, some research focused on mixing nanometer-sized particles or micrometer-sized conducting dust particles in the base fluid to solve the defect in the fluid. The base fluid will become nanofluid when it is permeated with nanometersized particles while it will become dusty fluid when it is permeated with dust particles. These methods help to enhance the heat transfer performance of the fluid. The study of dusty fluid

\footnotetext{
* Corresponding author.

E-mail address: u.wing0628@gmail.com

* Corresponding author.

E-mail address: syahira@uthm.edu.my
}

https://doi.org/10.37934/arfmts.89.2.4355 
becomes necessary because of its importance in the applications such as sedimentation, blood rheology and environmental pollution and flow through packed bed.

Saffman [1] was the first person who discuss the laminar flow of a dusty gas. Then, the characteristics of dusty gases at different conditions was analysed by Marble [2]. Madhura et al., [3] studied the motion of the unsteady dusty fluid through porous media in an open rectangle channel. The convective heat transfer characteristics of an incompressible dusty fluid over a vertical stretching sheet was observed by Gireesha et al., [4]. Sulochana and Sandeep [5] illustrated the flow of MHD dusty nanofluid toward a porous shrinking cylinder at different temperature. The natural convection flow of a two-phase dusty nanofluid along a vertical wavy frustum of a cone was examined by Siddiqa et al., [6]. The gyrotactic bioconvection of dusty nanofluid along a vertical isothermal surface by using numerical method was studied by Begum et al., [7]. Gireesha et al., [8] analysed the Hall effects on dusty nanofluid two-phase transient flow past a stretching sheet.

The convective heat transfer and flow characteristics of the nanofluid in a tube was considered by Li and Xuan [9]. Kwak and Kim [10] investigated the viscosity and thermal conductivity of copper oxide nanofluid for the dispersion in the ethyleneglycol. After that, Jang and Choi [11] carried out the influence of the various parameters on the thermal conductivity of the nanofluid. The MHD flow of a dusty fluid near the stagnation point over a permeable stretching sheet was studied by Ramesh et al., [12] by considering the effect of non-uniform source. Sandeep et al., [13] observed the unsteady MHD radiative flow of a dusty nanofluid over an exponentially pervious stretching surface. Kumar and Varma [14] published a study about the stagnation point flow of MHD nanofluid over a stretching surface through a porous medium in the presence of radiation and dissipation. The MHD Carreau dusty fluid over the stretching surface with exponentially decomposing heat source was illustrated by Mamatha et al., [15].

Soundalgekar and Ramana Murty [16] studied the boundary layer flow over a continuously moving plate. The boundary layer flow of a second-grade viscoelastic fluid over a continuously moving plate was explored by Sadeghy and Sharifi [17]. Then, Wang [18] presented the stagnation slip flow and heat transfer on a moving plate with partial slip condition. Najib [19] investigated the stability analysis for the flow and heat transfer over a moving plate. The problem of hydrodynamic and thermal boundary layers over a flat plate with convective boundary condition was explained by Aziz [20]. Rahman and Eltayeb [21] inspected the heat transfer of the hydromagnetic nanofluid over a non-linear stretching sheet with the effect of radiation and convective boundary condition. The study of stagnation point of hydromagnetic flow of dusty fluid toward stretching sheet heated by convection was analysed by Isa et al., [22].

The MHD flow of a double stratified micropolar fluid across a vertical shrinking sheet in the presence of suction, heat source effects and chemical reaction was analysed by Khan et al., [23]. Zokri et al., [24] explored the influence of viscous dissipation and suspended nanoparticles on mixed convection flow of Jeffrey fluid from a horizontal circular cylinder. Moreover, a Bingham nonNewtonian fluid flow in Forchheimer porous medium subjected to Hall current was modified by Ewis [25]. Ferdows et al., [26] investigated steady free convective boundary layer viscous fluid flow and heat transfer towards the moving flat plate subjected to suction and injection effects.

All the references above studied either dusty or nanofluid flows through different channels. However, no study has been done on the flow and heat transfer characteristics of MHD dusty nanofluid over the moving plate by considering convective boundary condition. Therefore, MHD flow of dusty nanofluid toward moving plate with convective boundary condition is studied. In this research, the governing equations of the flow and heat transfer are considered. Then, the partial differential governing equations will be transformed into a system of nonlinear ordinary differential equations using similarity transformation. At that point, the numerical solutions to the nonlinear 
ordinary differential equations will be obtained by using shooting method with fourth-fifth order Runge-Kutta Fehlberg method (RKF45).

\section{Methodology}

\subsection{Flow Analysis}

Consider a two dimensional laminar, incompressible and electrically conducting boundary layer flow toward a moving plate in the dusty nanofluid as shown in Figure 1. The flow is generated by two equal and opposite forces along the $x$-axis and $y$-axis that is normal to it. The plate (situated at $y=0$ ) is moving with the constant velocity $U_{w}$ and $U_{\infty}$ be the fluid velocity outside the boundary layer. The flow field is exposed to the influence of external magnetic field strength $B_{0}$ along $x$-axis. The fluid and dust particle clouds here supposed to be static at the beginning. The dust particles are assumed to have uniform size. Spherical shaped nano and dust particles are considered. The number density of dust particles along with volume fraction is taken into account. Then, the number density of dust particles is assumed to be constant throughout the flow. It is assumed that the external electric field due to the polarization of charge is negligible. The drag force is taken into account for fluid and particle interaction. Under the above assumptions by Sandeep and Saleem [27] and Bachok et al., [28], the boundary layer equations that governs the flow are given by

$$
\begin{aligned}
& \frac{\partial u}{\partial x}+\frac{\partial v}{\partial y}=0 \\
& \rho_{n f}\left(1-\phi_{p}\right)\left(u \frac{\partial u}{\partial x}+v \frac{\partial u}{\partial y}\right)=\left(1-\phi_{p}\right) \mu_{n f} \frac{\partial^{2} u}{\partial y^{2}}+K N\left(u_{p}-u\right)-\sigma B_{0}^{2} u \\
& u_{p} \frac{\partial u_{p}}{\partial x}+v_{p} \frac{\partial u_{p}}{\partial y}=\frac{K}{m}\left(u-u_{p}\right) \\
& \frac{\partial u_{p}}{\partial x}+\frac{\partial v_{p}}{\partial y}=0
\end{aligned}
$$

with boundary conditions

$$
\begin{aligned}
& u=U_{w}, v=0 \text { at } y=0 \\
& u \rightarrow U_{\infty}, u_{p} \rightarrow U_{\infty}, v_{p} \rightarrow v \text { as } y \rightarrow \infty
\end{aligned}
$$

where $(u, v)$ and $\left(u_{p}, v_{p}\right)$ are velocity components of the nanofluid and dust phase in the $x$ and $y$ direction respectively, $\phi_{p}$ is the volume fraction of the dust particle, $\mu_{n f}$ is the kinematic viscosity of the nanofluid, $K$ is the stokes resistance, $m$ is the mass of the dust particle, $N$ is the number density of the dust particles, $\rho_{n f}$ is the density of the nanofluid, $\sigma$ and $B_{0}$ are the electrical conductivity, induced magnetic field respectively. The nanofluid constants are

$$
\rho_{n f}=(1-\phi) \rho_{f}+\phi \rho_{s}
$$




$$
\begin{aligned}
& \mu_{n f}=\frac{\mu_{f}}{(1-\phi)^{2.5}} \\
& \left(\rho c_{p}\right)_{n f}=(1-\phi)\left(\rho c_{p}\right)_{f}+\phi\left(\rho c_{p}\right)_{s} \\
& \frac{k_{n f}}{k_{f}}=\frac{\left(k_{s}+2 k_{f}\right)-2 \phi\left(k_{f}-k_{s}\right)}{\left(k_{s}+2 k_{f}\right)+\phi\left(k_{f}-k_{s}\right)}
\end{aligned}
$$

where $\phi$ is the volume fraction of the nanoparticle, the subscripts $n f, f$ and $s$ refer to the properties of nanofluid, fluid and solid respectively [29].

For similarity solution, the similarity variables are introduced as follow

$$
\begin{aligned}
& \eta=y\left(\frac{U}{v_{f} x}\right)^{1 / 2} \\
& \psi=\left(v_{f} x U\right)^{1 / 2} f(\eta) \\
& \psi_{p}=\left(v_{f} x U\right)^{1 / 2} F(\eta)
\end{aligned}
$$

where $U$ is the composite velocity defined as $U=U_{w}+U_{\infty}$ [30]. $\psi$ is the stream function defined as $u=\partial \psi / \partial y$ and $v=-\partial \psi / \partial x$, which identically satisfies Eq. (1) to Eq. (4). By applying Eq. (10) Eq. (12), Eq. (2) and Eq. (3) are reduced into

$$
\begin{aligned}
& \frac{\left(1-\phi_{p}\right)}{(1-\phi)^{2.5}} f^{\prime \prime \prime}+\frac{1}{2}\left(1-\phi_{p}\right)\left[1-\phi+\phi\left(\frac{\rho_{s}}{\rho_{f}}\right)\right] f f^{\prime \prime}+\alpha \beta\left(F^{\prime}-f^{\prime}\right)-M f^{\prime}=0 \\
& F F^{\prime \prime}-2 \beta\left(F^{\prime}-f^{\prime}\right)=0
\end{aligned}
$$

with the transformed boundary conditions

$$
\begin{aligned}
& f^{\prime}(\eta)=\lambda, f(\eta)=0 \text { at } \eta=0 \\
& f^{\prime}(\eta) \rightarrow 1-\lambda, F^{\prime}(\eta) \rightarrow 1-\lambda, F(\eta) \rightarrow f(\eta) \text { as } \eta \rightarrow \infty
\end{aligned}
$$

where $\alpha=N m / \rho_{f}$ is the mass concentration of the dust particle, $\beta=K x / m U$ is the fluid particle interaction parameter for velocity, $M=\sigma B_{0}{ }^{2} x / \rho_{f} U$ is the magnetic field parameter and $\lambda=U_{w} / U$ is the velocity ratio parameter. The case $0<\lambda<1$ is when the plate and the fluid move in the same direction, but when $\lambda<0$ and $\lambda>1$, they move in the opposite direction. If $\lambda<0$, the free stream is directed toward the positive $x$-axis, while the plate moves toward the negative $x$-axis. If $\lambda>1$, the free stream is directed toward the negative $x$-axis, while the plate moves toward the positive $x$-axis. 


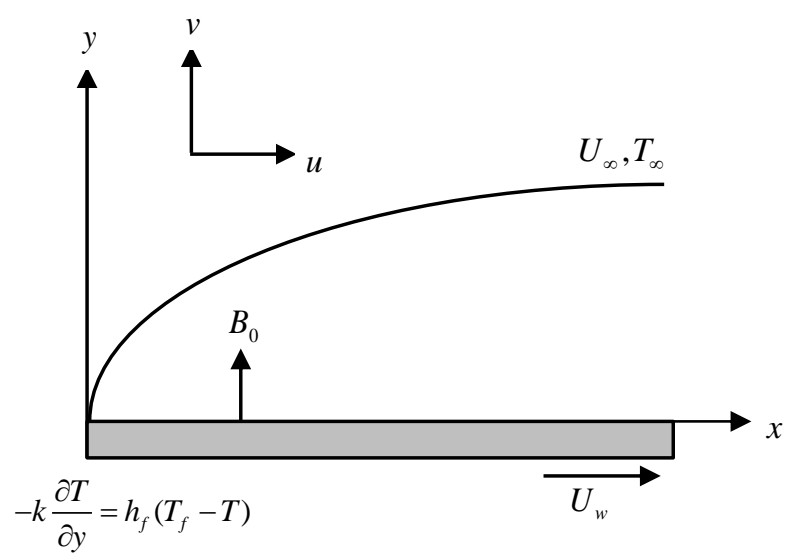

Fig. 1. Schematic diagram of the two dimensional boundary layer

\subsection{Heat Transfer Analysis}

The governing boundary layer heat transport equations for dusty nanofluid are

$$
\begin{aligned}
& \left(\rho c_{p}\right)_{n f}\left(u \frac{\partial T}{\partial x}+v \frac{\partial T}{\partial y}\right)=k_{n f} \frac{\partial^{2} T}{\partial y^{2}}+\frac{N_{1}\left(c_{p}\right)_{f}}{\tau_{T}}\left(T_{p}-T\right)+\frac{N_{1}}{\tau_{v}}\left(u_{p}-u\right)^{2} \\
& N_{1} c_{m}\left(u_{p} \frac{\partial T_{p}}{\partial x}+v_{p} \frac{\partial T_{p}}{\partial y}\right)=-\frac{N_{1}\left(c_{p}\right)_{f}}{\tau_{T}}\left(T_{p}-T\right)
\end{aligned}
$$

where $T$ and $T_{p}$ are the temperature of the nanofluid and dust particles respectively, $k_{n f}$ is the effective thermal conductivity of the nanofluid, $\left(c_{p}\right)_{f}, c_{m}$ are the specific heat of the fluid and dust particles respectively, $N_{1}=N m$ is the density of the particle phase, $\tau_{T}$ is the thermal equilibrium time, $\tau_{V}=m / K$ is the relaxation time of the dust particle. Then, the temperature boundary conditions are considered in order to solve Eq. (16) and Eq. (17) as

$$
\begin{aligned}
& -k \frac{\partial T}{\partial y}=h_{f}\left(T_{f}-T\right) \text { at } y=0 \\
& T \rightarrow T_{\infty}, T_{p} \rightarrow T_{\infty} \text { as } y \rightarrow \infty
\end{aligned}
$$

where $T_{f}$ is the hot fluid temperature and $h_{f}$ is the heat transfer coefficient.

To obtain the similarity solutions of Eq. (16) and Eq. (17), the non-dimensional variables are introduced as follow

$$
\theta(\eta)=\frac{T-T_{\infty}}{T_{f}-T_{\infty}}
$$




$$
\theta_{p}(\eta)=\frac{T_{p}-T_{\infty}}{T_{f}-T_{\infty}}
$$

Using Eq. (19) and Eq. (20) in Eq. (16) and Eq. (17), the nonlinear ordinary differential equations are obtained as

$$
\begin{aligned}
& \frac{1}{\operatorname{Pr}}\left[\frac{k_{n f} / k_{f}}{1-\phi+\phi\left(\rho c_{p}\right)_{s} /\left(\rho c_{p}\right)_{f}}\right] \theta^{\prime \prime}+\frac{1}{2} f \theta^{\prime}+\left[\frac{1}{1-\phi+\phi\left(\rho c_{p}\right)_{s} /\left(\rho c_{p}\right)_{f}}\right]\left[\alpha \beta_{T}\left(\theta_{p}-\theta\right)+\operatorname{Ec} \alpha \beta\left(F^{\prime}-f^{\prime}\right)^{2}\right]=0 \\
& F \theta_{p}{ }^{\prime}-2 \gamma \beta_{T}\left(\theta_{p}-\theta\right)=0
\end{aligned}
$$

subjected to the transformed boundary conditions

$$
\begin{aligned}
& \theta^{\prime}(\eta)=B i[\theta(\eta)-1] \text { at } \eta=0 \\
& \theta(\eta) \rightarrow 0, \theta_{p}(\eta) \rightarrow 0 \text { as } \eta \rightarrow \infty
\end{aligned}
$$

where $\operatorname{Pr}=\mu_{n f}\left(c_{p}\right)_{f} / k_{n f}$ is the Prandtl number, $\beta_{T}=x / \tau_{T} U$ is the fluid particle interaction parameter for temperature, $\mathrm{Ec}=U^{2} /\left(c_{p}\right)_{f}\left(T_{f}-T_{\infty}\right)$ is the Eckert number, $\gamma=\left(c_{p}\right)_{f} / c_{m}$ is the ratio of the specific heat of the nanofluid to the dust particles and $B i=\left(h_{f} / k\right)(v x / U)^{1 / 2}$ is the Biot number.

For the engineering interest, the skin friction coefficient $C_{f}$ and the local Nusselt number $N u_{x}$ are defined as

$$
C_{f}=\frac{\tau_{w}}{\rho_{n f} U^{2}} \quad N u_{x}=\frac{x q_{w}}{k_{n f}\left(T_{w}-T_{\infty}\right)}
$$

where the surface shear stress ${ }^{\tau_{w}}$ and the surface heat flux ${ }^{q_{w}}$ are given by

$$
\tau_{w}=\mu_{n f}\left(\frac{\partial u}{\partial y}\right)_{y=0} q_{w}=-k_{n f}\left(\frac{\partial T}{\partial y}\right)_{y=0}
$$

Using Eq. (10), Eq. (11), Eq. (12), Eq. (19) and Eq. (20), we get

$$
C_{f} \operatorname{Re}_{x}^{1 / 2}=\frac{1}{(1-\varphi)^{2.5}} f^{\prime \prime}(0) \quad N u_{x} \operatorname{Re}_{x}^{1 / 2}=-\frac{k_{n f}}{k_{f}} \theta^{\prime}(0)
$$

where $R e_{x}=U x / v_{f}$ is the local Reylonds number. 


\section{Results and Discussion}

The system of coupled ordinary differential equations as in Eq. (13), Eq. (14), Eq. (21) and Eq. (22) subject to boundary conditions (15) and (23) are solved by using shooting method with RKF45. The numerical solutions are obtained to study the effects of non-dimensional governing parameters such as velocity ratio parameter $\lambda$, magnetic field parameter $M$, volume fraction of the nanoparticle $\phi$, volume fraction of the dust particle $\phi_{p}$, mass concentration of the dust particle $\alpha$, fluid particle interaction parameter for velocity $\beta$, fluid particle interaction parameter for temperature $\beta_{T}$ and Biot number $B i$ on the velocity and temperature profiles for fluid and dust phases of CuO-water and $\mathrm{Al}_{2} \mathrm{O}_{3}$-water dusty nanofluids are presented graphically with $0 \leq \eta \leq 10$. For numerical results, the parameters are considered as $\lambda=M=1, \phi=\phi_{p}=0.1, \alpha=\beta_{T}=0.2, \beta=0.5, \operatorname{Pr}=1, \operatorname{Ec}=3$, $B i=10$ where these values are kept as common in this study except the varies values presented in figures. The thermophysical properties of water, Copper Oxide, Aluminium Oxide are given as shown in Table 1 [28].

\section{Table 1}

Thermophysical properties of fluid and nanoparticles

\begin{tabular}{llll}
\hline & $\rho\left(\mathrm{Kgm}^{-3}\right)$ & $k\left(\mathrm{Wm}^{-1} \mathrm{~K}^{-1}\right)$ & $c_{p}\left(\mathrm{JKg}^{-1} \mathrm{~K}^{-1}\right)$ \\
\hline $\mathrm{H}_{2} \mathrm{O}$ & 997.1 & 0.613 & 4179 \\
$\mathrm{CuO}$ & 6320 & 76.5 & 531.8 \\
$\mathrm{Al}_{2} \mathrm{O}_{3}$ & 3970 & 40 & 765 \\
\hline
\end{tabular}

Figure 2 and 3 display the velocity and temperature profiles of fluid and dust phases for various values of velocity ratio parameter $\lambda$. From Figure 2 , it is noticed that the velocity of the fluid and dust decrease when $\lambda \geq 1$. However, no solution exists when $\lambda<1$. Figure 3 shows that an increase in $\lambda$ enhances the temperature profiles of fluid but reduces the temperature of dust phase. Figure 4 and Figure 5 illustrate the velocity and temperature profiles of fluid and dust phases for various values of magnetic field parameter $M$. The results show that there is a depreciation on the velocity profiles and improvement on the temperature profiles for both phases when $M$ increases. An increase in $M$ generates the opposite force to the flow, which is Lorentz force. This force declines the velocity boundary layer and enhances the thermal boundary layer.

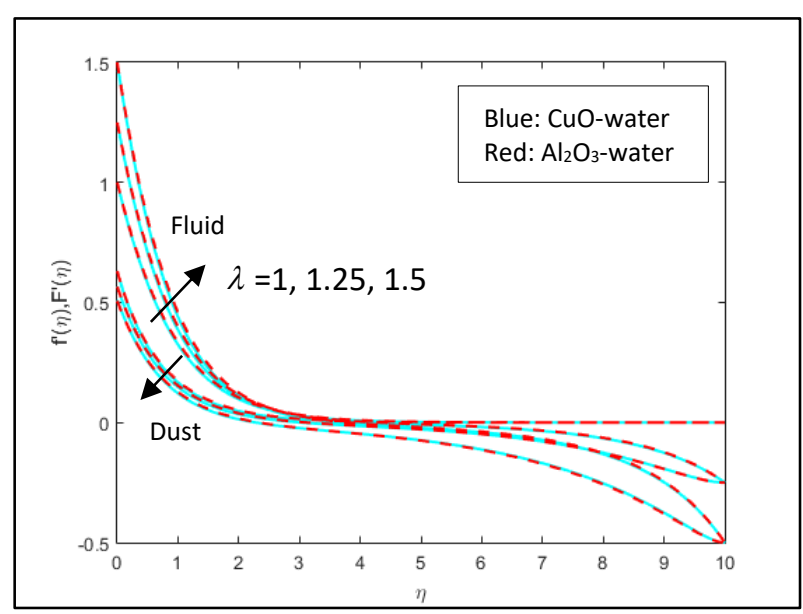

Fig. 2. Velocity profiles of fluid and dust phases for various value of $\lambda$

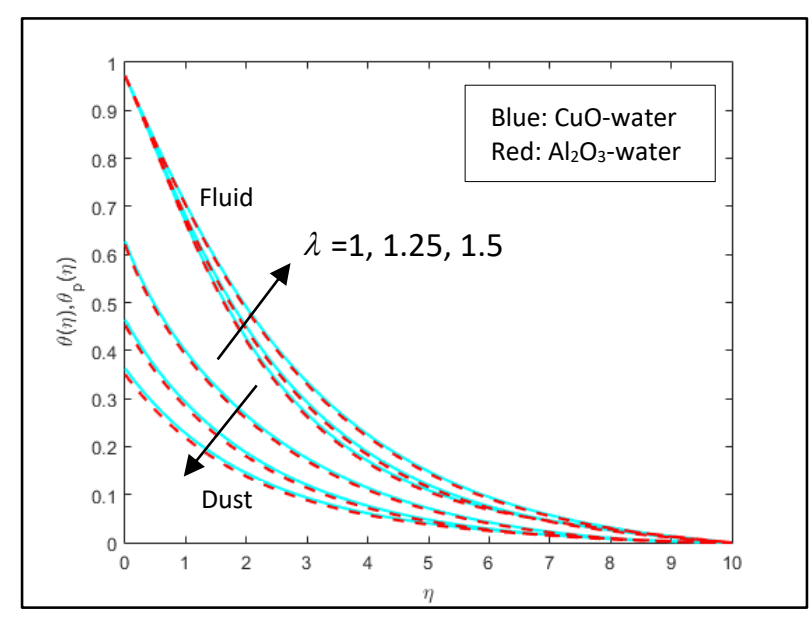

Fig. 3. Temperature profiles of fluid and dust phases for various value of $\lambda$ 


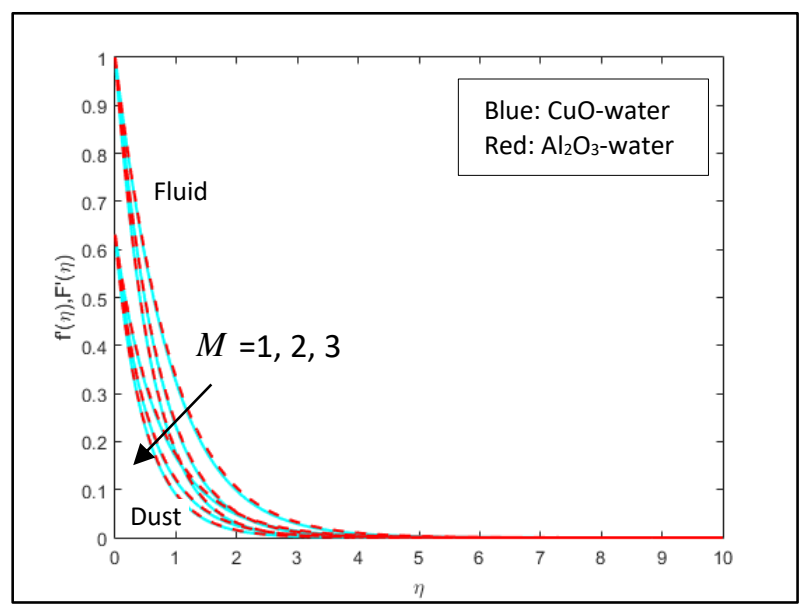

Fig. 4. Velocity profiles of fluid and dust phases for various value of $M$

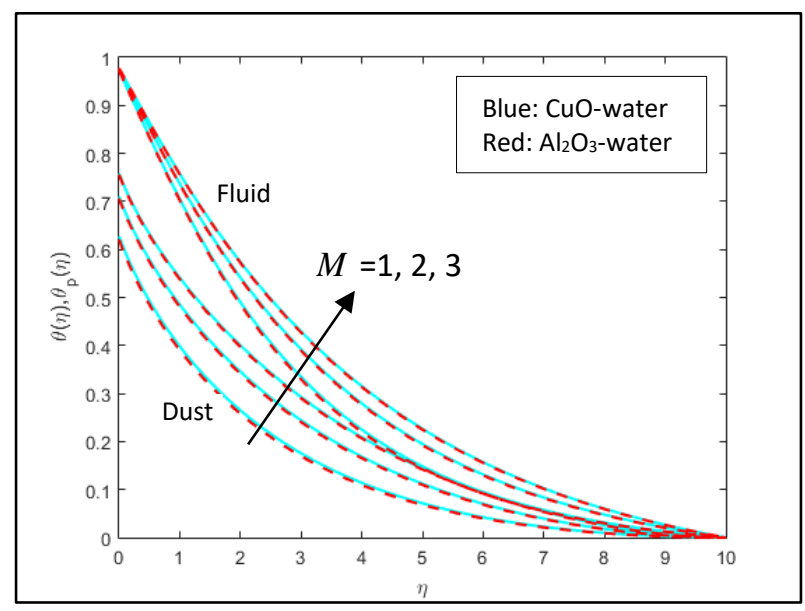

Fig. 5. Temperature profiles of fluid and dust phases for various value of $M$

Figure 6 and Figure 7 show the velocity and temperature profiles of fluid and dust phases for various values of volume fraction of the nanoparticle $\phi$. It is clearly seen that an increase in $\phi$ enhances both velocity and temperature of the fluid and dust phases. It is also noticed that the temperature profiles of dust phase has a very slight enhancement compared to fluid phase. This proved that the increasing values of $\phi$ has good interaction with the fluid particles than dust particles in enhancing the thermal conductivity of the fluid. Figure 8 and Figure 9 depict the velocity and temperature profiles of fluid and dust phases for various values of volume fraction of the dust particle $\phi_{p}$. It is observed that as $\phi_{p}$ increases, the velocity profiles decreases but the temperature profiles increases for both phases. This situation occurs due to the enhancement of the volume occupied by the dust particles when there is an increase in $\phi_{p}$ volume fraction of dust particle. These particles cause shear stress near the layers and depreciates the velocity profiles.

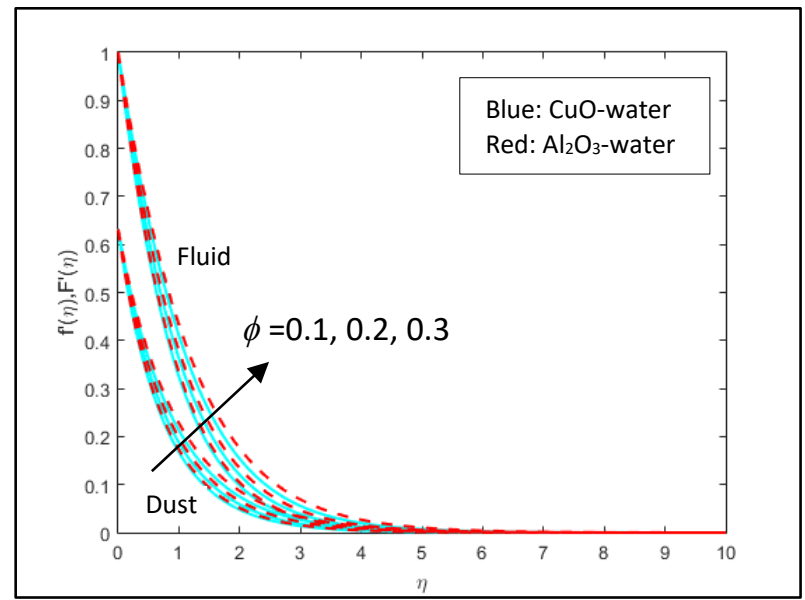

Fig. 6. Velocity profiles of fluid and dust phases for various value of $\phi$

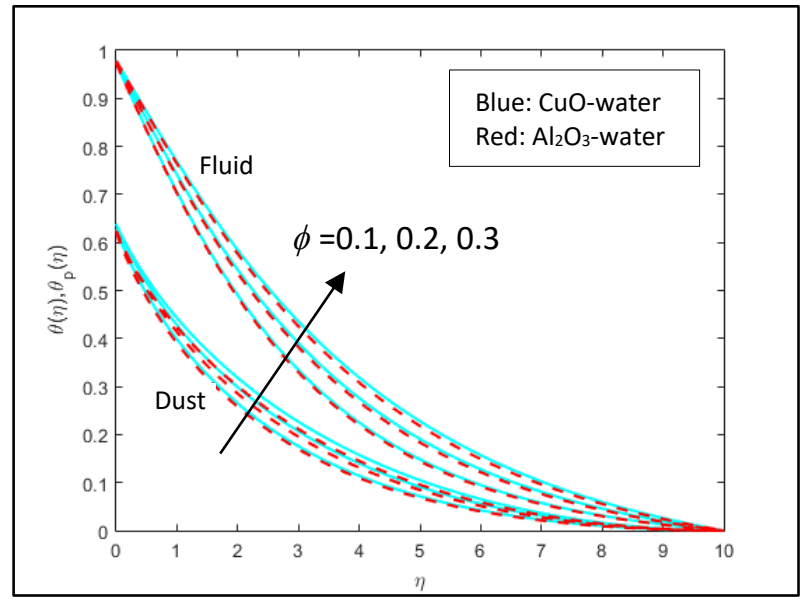

Fig. 7. Temperature profiles of fluid and dust phases for various value of $\phi$ 


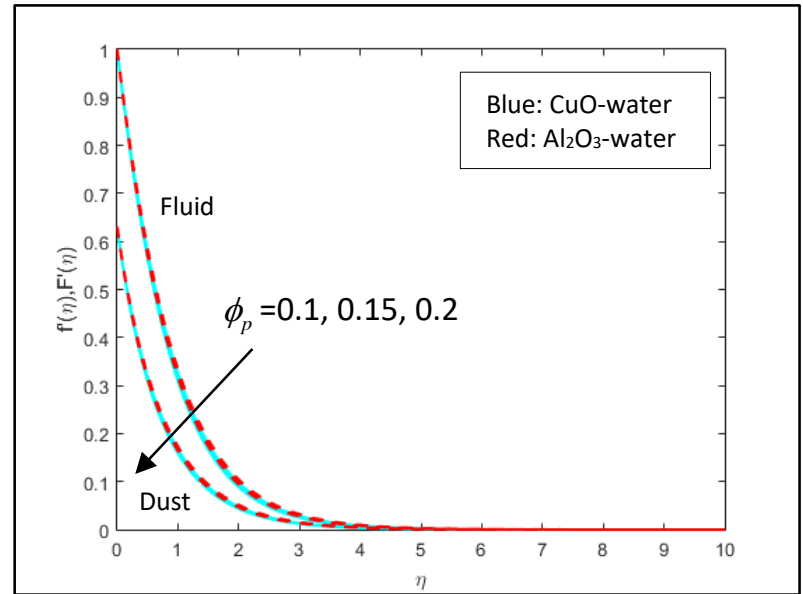

Fig. 8. Velocity profiles of fluid and dust phases for various value of $\phi_{p}$

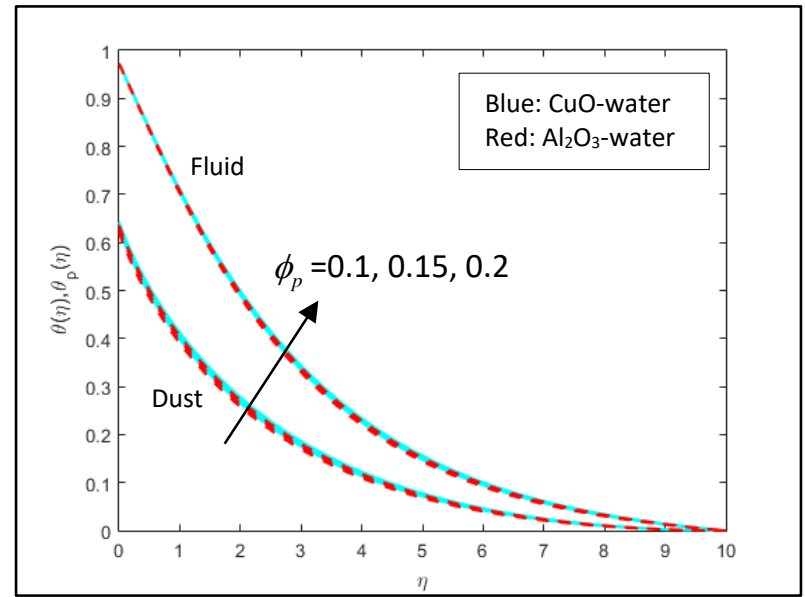

Fig. 9. Temperature profiles of fluid and dust phases for various value of $\phi_{p}$

The velocity and temperature profiles of fluid and dust phases for various values of mass concentration of dust particle $\alpha$ are displayed as shown in Figure 10 and Figure 11 . The results show that an enhancement of $\alpha$ declines both velocity and temperature profiles of both phases. This concluded that the increasing values of $\alpha$ reduces the boundary layers of velocity and temperature. Figure 12 and Figure 13 reveal the velocity and temperature profiles of fluid and dust phases for various values of fluid particle interaction parameter for velocity $\beta$. From Figure 12 , it observed that the velocity profiles of the dust phase enhanced but the velocity of the fluid phase depreciated when $\beta$ increases. This is due to the interaction between the fluid and particle phase is high and the development of the opposite force from the particle phase to the fluid phase happens until the particle velocity reaches the fluid velocity. From Figure 13, it is clear that the enhancement in $\beta$ increases the temperature profiles of the both phases. This verified that there is higher interaction between the fluid and particle phase and it will improve the thermal conductivity of the flow.

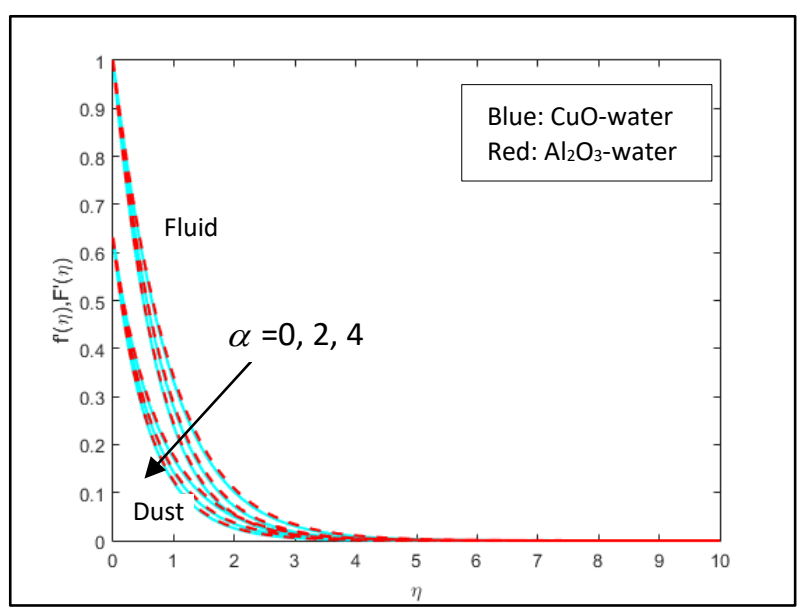

Fig. 10. Velocity profiles of fluid and dust phases for various value of $\alpha$

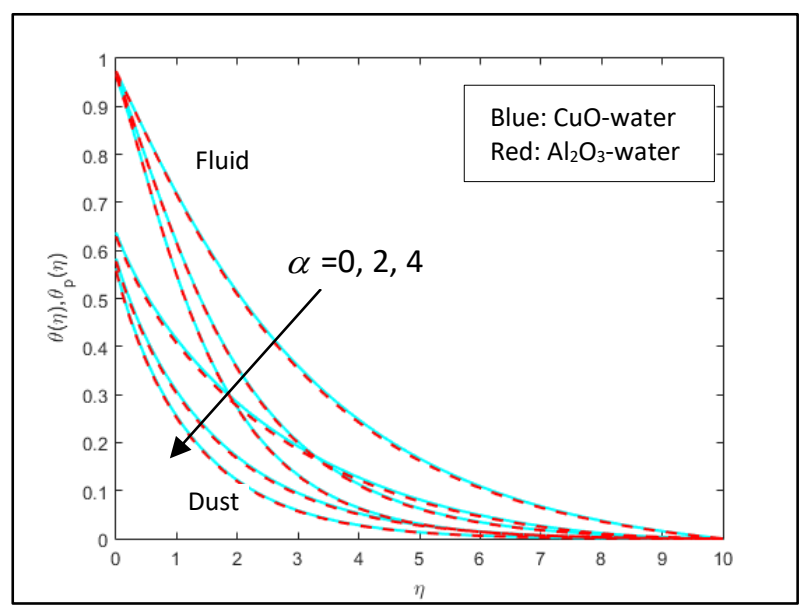

Fig. 11. Temperature profiles of fluid and dust phases for various value of $\alpha$ 


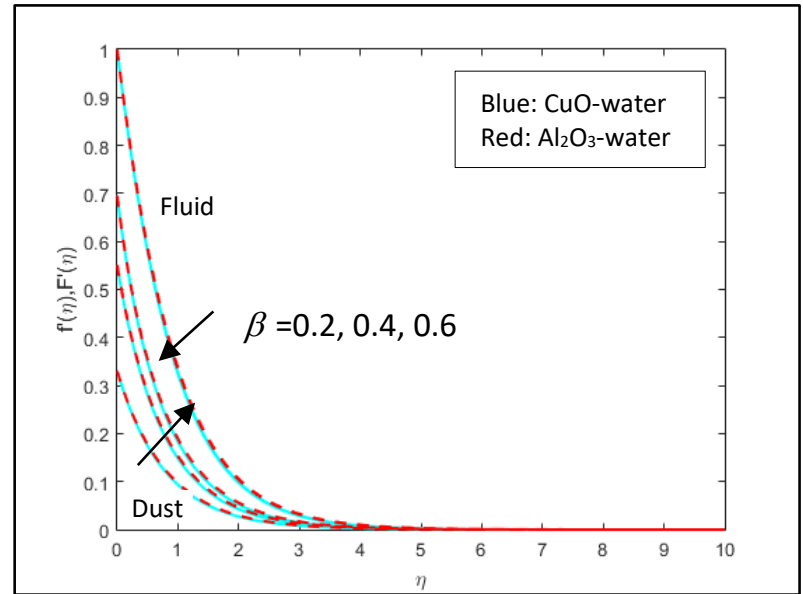

Fig. 12. Velocity profiles of fluid and dust phases for various value of $\beta$

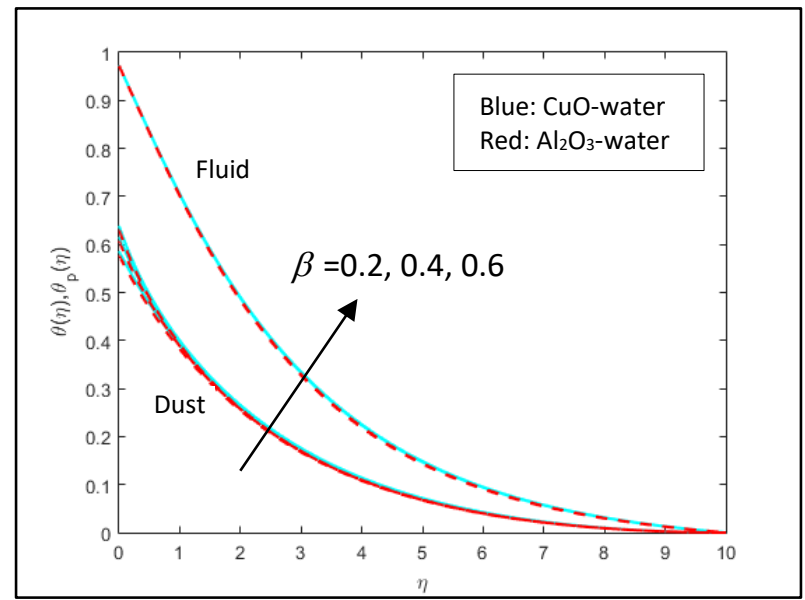

Fig. 13. Temperature profiles of fluid and dust phases for various value of $\beta$

Figure 14 depicts the temperature profiles of fluid and dust phases for various values of fluid particle interaction parameter for temperature $\beta_{T}$. It is evident that an increase in $\beta_{T}$ reduces the temperature of fluid phase but enhances the temperature of dust phase. This is because the effect of convective heat transfer in dust phase strengthen the interaction between the nanoparticle and dust particle phase. Figure 15 shows the temperature profiles of fluid and dust phases for various values of Biot number $B i$. It is obviously seen that the increasing of $B i$ increases the temperature profiles of both phases. This concluded that the effect of $B i$ is to increase the thermal boundary layer.

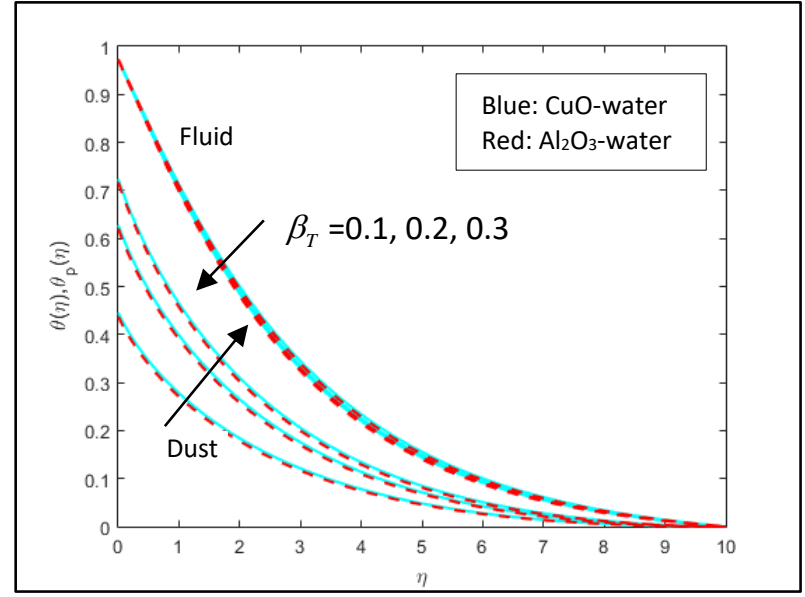

Fig. 14. Temperature profiles of fluid and dust phases for various value of $\beta_{T}$

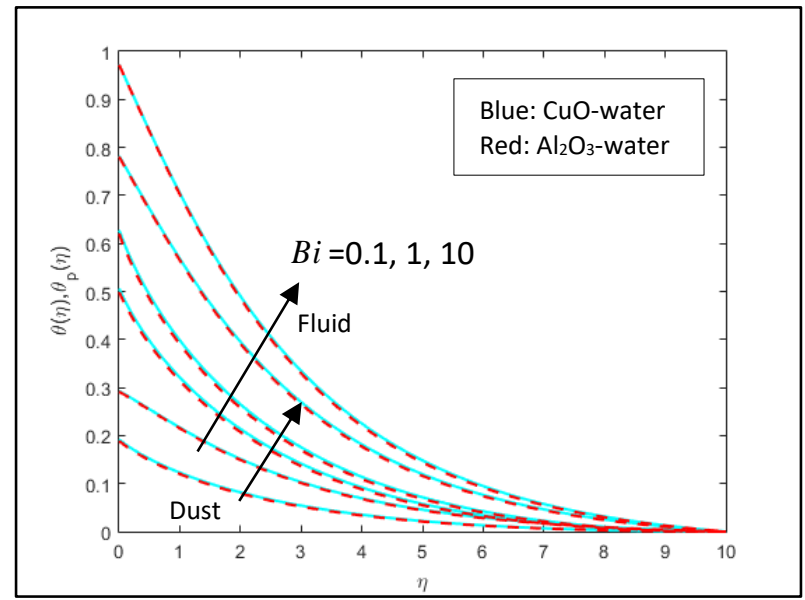

Fig. 15. Temperature profiles of fluid and dust phases for various value of $\mathrm{Bi}$

Table 2 and Table 3 represent the variation of non-dimensional governing parameters in skin friction coefficient and Nusselt number for $\mathrm{CuO}$-water and $\mathrm{Al}_{2} \mathrm{O}_{3}$-water dusty nanofluids. The results from Table 2 and Table 3 show that the increasing values of $M, \phi_{p}$ and $\beta$ increases the friction factor but depreciates heat transfer rate in both dusty nanofluids. An increase in $\phi$ shows a decrement of both friction factor and heat transfer rate. There is an increment of friction factor and the enhancement of heat transfer rate when $\alpha$ increases. Moreover, an increase in $\beta_{T}$ and $B i$ enhances the heat transfer rate, but it does not show any effect on the friction factor for both dusty nanofluids. 


\section{Table 2}

Variation in $f^{\prime \prime}(0)$ and $-\theta^{\prime}(0)$ for CuO-water dusty nanofluid

\begin{tabular}{|c|c|c|c|c|c|c|c|c|}
\hline$M$ & $\phi$ & $\phi_{p}$ & $\alpha$ & $\beta$ & $\beta_{T}$ & $B i$ & $\left|f^{\prime \prime}(0)\right|$ & $-\theta^{\prime}(0)$ \\
\hline 1 & 0.1 & 0.1 & 0.2 & 0.5 & 0.2 & 10 & 1.046877 & 0.274890 \\
\hline 2 & & & & & & & 1.394885 & 0.243871 \\
\hline 3 & & & & & & & 1.672692 & 0.224452 \\
\hline \multirow[t]{3}{*}{1} & 0.1 & 0.1 & 0.2 & 0.5 & 0.2 & 10 & 1.046877 & 0.274890 \\
\hline & 0.2 & & & & & & 0.933776 & 0.238998 \\
\hline & 0.3 & & & & & & 0.815324 & 0.212522 \\
\hline \multirow[t]{3}{*}{1} & 0.1 & 0.1 & 0.2 & 0.5 & 0.2 & 10 & 1.046877 & 0.274890 \\
\hline & & 0.15 & & & & & 1.071418 & 0.272428 \\
\hline & & 0.2 & & & & & 1.098388 & 0.269769 \\
\hline \multirow[t]{3}{*}{1} & 0.1 & 0.1 & 1 & 0.5 & 0.2 & 10 & 1.114823 & 0.300981 \\
\hline & & & 2 & & & & 1.194272 & 0.326121 \\
\hline & & & 3 & & & & 1.268711 & 0.346840 \\
\hline \multirow[t]{3}{*}{1} & 0.1 & 0.1 & 0.2 & 0.1 & 0.2 & 10 & 1.035974 & 0.281283 \\
\hline & & & & 0.2 & & & 1.040538 & 0.275579 \\
\hline & & & & 0.3 & & & 1.043582 & 0.273815 \\
\hline \multirow[t]{3}{*}{1} & 0.1 & 0.1 & 0.2 & 0.5 & 0.5 & 10 & 1.046877 & 0.283177 \\
\hline & & & & & 1 & & 1.046877 & 0.286726 \\
\hline & & & & & 1.5 & & 1.046877 & 0.287914 \\
\hline \multirow[t]{3}{*}{1} & 0.1 & 0.1 & 0.2 & 0.5 & 0.2 & 0.1 & 1.046877 & 0.070724 \\
\hline & & & & & & 10 & 1.046877 & 0.274890 \\
\hline & & & & & & 100 & 1.046877 & 0.282299 \\
\hline
\end{tabular}

\section{Table 3}

Variation in $f^{\prime \prime}(0)$ and $-\theta^{\prime}(0)$ for $\mathrm{Al}_{2} \mathrm{O}_{3}$-water dusty nanofluid

\begin{tabular}{|c|c|c|c|c|c|c|c|c|}
\hline$M$ & $\phi$ & $\phi_{p}$ & $\alpha$ & $\beta$ & $\beta_{T}$ & $B i$ & $\left|f^{\prime \prime}(0)\right|$ & $-\theta^{\prime}(0)$ \\
\hline 1 & 0.1 & 0.1 & 0.2 & 0.5 & 0.2 & 10 & 1.031226 & 0.277651 \\
\hline 2 & & & & & & & 1.383520 & 0.245375 \\
\hline 3 & & & & & & & 1.663349 & 0.225407 \\
\hline \multirow[t]{3}{*}{1} & 0.1 & 0.1 & 0.2 & 0.5 & 0.2 & 10 & 1.031226 & 0.277651 \\
\hline & 0.2 & & & & & & 0.907120 & 0.243031 \\
\hline & 0.3 & & & & & & 0.781982 & 0.216900 \\
\hline \multirow[t]{3}{*}{1} & 0.1 & 0.1 & 0.2 & 0.5 & 0.2 & 10 & 1.031226 & 0.277651 \\
\hline & & 0.15 & & & & & 1.056172 & 0.275069 \\
\hline & & 0.2 & & & & & 1.083563 & 0.272286 \\
\hline \multirow[t]{3}{*}{1} & 0.1 & 0.1 & 1 & 0.5 & 0.2 & 10 & 1.100101 & 0.303116 \\
\hline & & & 2 & & & & 1.180505 & 0.327678 \\
\hline & & & 3 & & & & 1.255733 & 0.347999 \\
\hline \multirow[t]{3}{*}{1} & 0.1 & 0.1 & 0.2 & 0.1 & 0.2 & 10 & 1.020175 & 0.284198 \\
\hline & & & & 0.2 & & & 1.024809 & 0.278355 \\
\hline & & & & 0.3 & & & 1.027894 & 0.276551 \\
\hline \multirow[t]{3}{*}{1} & 0.1 & 0.1 & 0.2 & 0.5 & 0.5 & 10 & 1.031226 & 0.286279 \\
\hline & & & & & 1 & & 1.031226 & 0.290019 \\
\hline & & & & & 1.5 & & 1.031226 & 0.291278 \\
\hline \multirow[t]{3}{*}{1} & 0.1 & 0.1 & 0.2 & 0.5 & 0.2 & 0.1 & 1.031226 & 0.070871 \\
\hline & & & & & & 10 & 1.031226 & 0.277651 \\
\hline & & & & & & 100 & 1.031226 & 0.285216 \\
\hline
\end{tabular}




\section{Conclusions}

The numerical solutions for analyzing the flow and heat transfer characteristics of MHD dusty nanofluid toward moving plate with convective boundary condition are discussed. CuO-water and $\mathrm{Al}_{2} \mathrm{O}_{3}$-water nanofluids permeated with dust particles are considered. The influence of nondimensional governing parameters on the velocity and temperature profiles of both fluid and dust phases are presented graphically. Then, skin friction coefficient and Nusselt number for CuO-water and $\mathrm{Al}_{2} \mathrm{O}_{3}$-water dusty nanofluids are discussed in tabular form. The conclusions of this study as follows

i. Magnetic field parameter has trend to develop opposite force to the flow and declines the velocity boundary layer and improves the thermal boundary layer.

ii. A raise in volume fraction of nano particle and volume fraction in dust particle enhances the temperature profiles of the flow. CuO-water dusty nanofluid has higher effect than $\mathrm{Al}_{2} \mathrm{O}_{3}-$ water dusty nanofluid in this condition.

iii. An increase in mass concentration of dust particle reduces the velocity and thermal boundary layer thickness.

iv. Fluid particle interaction parameter for velocity and temperature has trend to improve the temperature profiles of dust phase.

v. An increase in Biot number enhances temperature profiles of the flow and heat transfer rate.

\section{Acknowledgement}

The authors are grateful to Research Management Centre (RMC) of Universiti Tun Hussein Onn Malaysia (Project Code: GPPS-H634) and (Project Code: TIER 1-H096) for the financial support.

\section{References}

[1] Saffman, P.G. "On the stability of laminar flow of a dusty gas." Journal of Fluid Mechanics 13, no. 1 (1962): $120-128$. https://doi.org/10.1017/S0022112062000555

[2] Marble, F.E. "Dynamics of dusty gases." Annual Review of Fluid Mechanics 2 (1970): $397-446$. https://doi.org/10.1146/annurev.fl.02.010170.002145

[3] Madhura, K.R., B.J. Gireesha, and C.S. Bagewadi. "Exact solutions of unsteady dusty fluid flow through porous media in an open rectangular channel." Advances in Theoretical and Applied Mechanics 2, no. 1 (2009): 1-17.

[4] Gireesha, B.J., G.K. Ramesh, H.J. Lokesh, and C.S. Bagewadi. "Boundary layer flow and heat transfer of a dusty fluid over a stretching vertical surface." Applied Mathematics 2, no. $4 \quad$ (2011): $475-481$. https://doi.org/10.4236/am.2011.24061

[5] Sulochana, C. and N. Sandeep. "Flow and heat transfer behavior of MHD dusty nanofluid past a porous stretching/shrinking cylinder at different temperatures." Journal of Applied Fluid Mechanics 9, no. 2 (2015): 543553. https://doi.org/10.18869/acadpub.jafm.68.225.24847

[6] Siddiqa, S., N. Begum, M.A. Hossain, and R.S.R. Gorla. "Numerical solutions of natural convection flow of a dusty nanofluid about a vertical wavy truncated cone." Journal of Heat Transfer 139, no. 2 (2016): 022553. https://doi.org/10.1115/1.4034815

[7] Begum, N., S. Siddiqa, M. Sulaiman, S. Islam, M.A. Hossain, and R.S.R. Gorla. "Numerical solutions for gyrotactic bioconvection of dusty nanofluid along a vertical isothermal surface." International Journal of Heat and Mass Transfer 113 (2017): 229-236. https://doi.org/10.1016/j.ijheatmasstransfer.2017.05.071

[8] Gireesha, B.J., B. Mahanthesh, G.T. Thammanna, and P.B. Sampathkumar. "Hall effects on dusty nanofluid twophase transient flow past a stretching sheet using KVL Model." Journal of Molecular Liquids 256 (2018): $139-147$. https://doi.org/10.1016/i.molliq.2018.01.186

[9] Li, Q. and Y. Xuan. "Convective heat transfer and flow characteristics of Cu-water nanofluid." Science in China Series E: Technolgical Science 45, no. 4 (2002).

[10] Kwak, K. and C. Kim. "Viscosity of thermal conductivity of Copper Oxide nanofluid dispersed in Ethylene Glycol." Korea-Australia Rheology Journal 17, no. 2 (2005): 35-40. 
[11] Jang, S.P. and S.U.S. Choi. "Effects of various parameters on nanofluid thermal conductivity." Journal of Heat Transfer 129, no. 5 (2007): 617-623. https://doi.org/10.1115/1.2712475

[12] Ramesh, G.K., B.J. Gireesha, and C.S. Bagewadi. "MHD flow of a dusty fluid near the stagnation point over a permeable stretching sheet with non-uniform source/sink." International Journal of Heat and Mass Transfer 55, no. 17-18 (2012): 4900-4907. https://doi.org/10.1016/j.ijheatmasstransfer.2012.05.003

[13] Sandeep, N., C. Sulochana, and B.R. Kumar. "Unsteady MHD radiative flow and heat transfer of a dusty nanofluid over an exponentially stretching surface." Engineering Science and Technology, An International Journal 19, no. 1 (2016): 227-240. https://doi.org/10.1016/i.jestch.2015.06.004

[14] KiranKumar, R.V.M.S.S. and S.V.K. Varma. "Stagnation point flow of thermally radiated and dissipative MHD nanofluid over a stretching sheet filled with porous medium and suction." Songklanakarin Journal of Science and Technology 41, no. 1 (2017): 123-135.

[15] Mamatha, S.U., Mahesha, C.S.K. Raju, and O.D. Makinde. "Effect of convective boundary conditions on MHD Carreau dusty fluid over a stretching sheet with heat source." Defect and Diffusion Forum 377 (2017): $233-241$. https://doi.org/10.4028/www.scientific.net/DDF.377.233

[16] Soundalgekar, V.M. and T.V.R. Murty. "Heat transfer in flow past a continuous moving plate with variable temperature." Wärme-und Stoffübertragung 14, no. 2 (1980): 91-93. https://doi.org/10.1007/BF01806474

[17] Sadeghy, K. and M. Sharifi. "Local similarity solution for the flow of a "Second-Grade" viscoelastic fluid above a moving plate." International Journal of Non-Linear Mechanics 39, no. 8 (2004): 1265-1273. https://doi.org/10.1016/j.ijnonlinmec.2003.08.005

[18] Wang, S.Y. "Stagnation slip flow and heat transfer on a moving plate." Chemical Engineering Science 61, no. 23 (2006): 7668-7672. https://doi.org/10.1016/i.ces.2006.09.003

[19] Najib, N., N. Bachok, N.M. Arifin, and N. Senu. "Boundary layer flow and heat transfer of nanofluids over a moving plate with partial slip and thermal convective boundary condition: Stability analysis." International Journal of Mechanics 11 (2017): 18-24.

[20] Aziz, A. "A similarity solution for laminar thermal boundary layer over a flat plate with a convective surface boundary condition." Communications in Nonlinear Science and Numerical Simulation 14, no. 4 (2009): 1064-1068. https://doi.org/10.1016/i.cnsns.2008.05.003

[21] Rahman, M.M. and I.A. Eltayeb. "Radiative heat transfer in a hydromagnetic nanofluid past a non-linear stretching surface with convective boundary condition." Meccanica 48, no. 3 (2012): 601-615. https://doi.org/10.1007/s11012-012-9618-2

[22] Isa, S.M., A. Ali, and S. Shafie. "Stagnation point flow of MHD dusty fluid toward stretching sheet with convective surface." Jurnal Teknologi (Sciences \& Engineering) 72, no. 1 (2014): 1-6.

[23] Khan, A.A., K. Zaimi, S.F. Sufahani and M. Ferdows. "MHD flow and heat transfer of double stratified micropolar fluid over a vertical permeable shrinking/stretching sheet with chemical reaction and heat source." Journal of Advanced Research in Applied Sciences and Engineering Technology 21, no. 1 (2020): 1-14. https://doi.org/10.37934/araset.21.1.114

[24] Zokri, S.M, N.S. Arifin, A.R.M. Kasim and M.Z. Salleh. "Free convection boundary layer flow of Jeffrey nanofluid on a horizontal circular cylinder with viscous dissipation effect." Journal of Advanced Research in Micro and Nano Engineering 1, no. 1 (2020): 1-14.

[25] Ewis, K.M. "Analytical solution of modified Bingham fluid flow through parallel plates channel subjected to Forchheimer medium and hall current using Linearized Differential Transformation Method." Journal of Advanced Research in Numerical Heat Transfer 4, no. 1 (2021): 14-31.

[26] Ferdows, M., M. Shamshuddin and K. Zaimi. "Computation of steady free convective boundary layer viscous fluid flow and heat transfer towards the moving flat subjected to suction/injection effects." CFD Letters 13, no. 3 (2021): 16-24. https://doi.org/10.37934/cfdl.13.3.1624

[27] Sandeep, N. and S. Saleem. "MHD flow and heat transfer of a dusty nano fluid over a stretching surface in porous medium." Jorden Journal of Civil Engineering 11, no. 4 (2015): 149-164.

[28] Bachok, N., A. Ishak and I. Pop. "Flow and heat transfer characteristics on a moving plate in a nanofluid." International Journal of Heat and Mass Transfer 55, no. 4 (2012): 642-648. https://doi.org/10.1016/j.ijheatmasstransfer.2011.10.047

[29] Oztop, H.F. and E. Abu-Nada. "Numerical study of natural convection in partially heated rectangular enclosures filled with nanofluids." International Journal of Heat and Mass Transfer 29, no. 5 (2008): 1326-1336. https://doi.org/10.1016/j.ijheatfluidflow.2008.04.009

[30] Afzal, N., A. Badaruddin, and A. Elgarvi. "Momentum and heat transport on a continuous flat surface moving in a parallel stream." International Journal of Heat and Mass Transfer 36, no. 13 (1993): 3399-3403. https://doi.org/10.1016/0017-9310(93)90022-X 